DOI: $10.3901 / J M E .2013 .17 .026$

\title{
微圆弧刀金刚石车刀在线图像测量方法*
}

\author{
仇中军 ${ }^{1}$ 房丰洲 $^{1}$ 赵群章 $^{1}$ 周立波 ${ }^{2}$ \\ (1. 天津大学精密测试技术及仪器国家重点实验室 天津 300072; \\ 2. 日本茨城大学智能系统工程系 日立 316-8511 日本)
}

\begin{abstract}
摘要: 为摆脱传统圆弧刃金刚石车刀制造对操作者技能熟练程度和经验的依赖, 并克服制造过程中需反复离线测量的缺点, 利用在线图像测量方法代替传统制造方法中的离线测量, 将获取的切削刃的形状信息和位置信息实时反馈给控制系统, 直接 驱动研磨系统带动金刚石车刀按照最优的加工策略针对性的进行切削刃轮廓自动研磨和修整。通过比较三种车刀切削刃圆弧 获取方法的效率及精确性, 确定获取金刚石车刀切削刃圆弧中心及研磨系统的旋转中心的最佳图像处理方法。利用在线获取 金刚石车刀切削刃圆弧中心及实时计算其和研磨系统的旋转中心距离, 实现初始装夹时金刚石车刀位置的实时调整, 从而消 除初始安装误差切削刃加工精度及效率的影响。开发适用于微圆弧刃金刚石车刀测量的图像测量系统, 实际测量验证试验表 明, 利用开发的在线图像测量方法获得的切削刃轮廓信息从原理上更准确，从而为实现圆弧刃金刚石车刀高效、高精度、低 成本的稳定加工提供了保证。
\end{abstract}

关键词: 金刚石车刀 图像处理 在线测量 研磨

中图分类号: TG712

\section{Online Image Measurement Method for Micro-round Nose Diamond Turning Tools}

\section{QIU Zhongjun ${ }^{1}$ FANG Fengzhou ${ }^{1}$ ZHAO Qunzhang ${ }^{1}$ ZHOU Libo ${ }^{2}$}

(1. State key Laboratory of Precision Measuring Technology and Instruments, Tianjin University, Tianjin 300072;

2. Department of Intelligent System Engineering, Ibaraki University, Hitachi 316-8511, Japan)

\begin{abstract}
In order to overcome the constraints of the traditional manufacturing method of diamond turning tool that the fabrication process relies on the operator's skill and experiences and needs repeated offline measurements, an online image measurement method is used to replace the offline measurement in the traditional manufacturing method. According to the information of cutting edge shape and location, the grinding system automatically drives the diamond turning tool to grind the edge contour pertinently through optimal processing strategies. The optimal image processing methods for obtaining the center of diamond turning tool cutting edge and center of rotation of the grinding system are determined through a comparison of the efficiency and accuracy of the three methods for obtaining tool cutting edge. By adjusting the distance between the center of diamond turning tool cutting edge and center of rotation of the grinding system on real time, the initial clamping error which influences the precision and efficiency of cutting edge processing is eliminated. An image measurement system suitable for measuring micro-round nose diamond turning tool is developed. The experiments show that the obtained information of cutting edge contour using the developed online image measurement system is more accurate, accurate, and the highly efficiency, high precision, low-cost and stable manufacturing of micro-round nose diamond turning tool is guaranteed.
\end{abstract}

Key words: Diamond turning tool Image processing Online measurement Grinding

\section{0 前言}

目前，使用圆弧刃单晶金刚石车刀进行超精密

\footnotetext{
*国家自然科学基金(50905125)、国家高技术研究发展计划(863 计划, 2012AA040405)和天津市自然科学基金(12JCZDJC27900)资助项目。 20121012 收到初稿, 20130420 收到修改稿
}

车削的方法被认为是实现复杂形状加工的最佳方法 之一 ${ }^{[1-4]}$ 。单晶金刚石具有摩擦因数低、导热率高、 硬度高以及可加工出接近原子尺寸级的锋利刃口等 特点, 是理想的复杂型面超精密加工刀具材料 ${ }^{[5-6]}$ 。 和直线刃车刀相比, 圆弧刃金刚石车刀在进行复杂 型面微细切削过程中对刀容易, 更易形成对切屑、 
切削力的稳定控制, 能够获得较高的复杂曲面形状 精度 ${ }^{[7-8]}$, 因此高精度微圆弧刃金刚石车刀的市场需 求不断扩大。但是单晶金刚石属于立方晶系，其外 观型面多表现为正六面体或者正十二面体，车刀制 造过程中仅靠研磨金刚石刀具毛坏前、后刀面很难 形成高精度圆弧切削刃，而且微观上单晶金刚石表 现为每个晶面上原子排列形式、原子密度以及各晶 面之间的距离都不同，从而形成天然金刚石晶体的 各向异性, 使切削刃不同处位置在相同研磨工艺下 材料去除难易程度不同, 造成了车刀切削刃的刃型

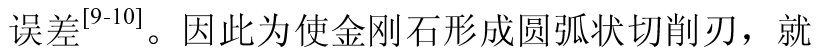
必须要对切削刃形状进行有针对性的圆弧修整加 工, 以确保金刚石车刀切削刃的轮廓度符合超精密 加工要求。

目前，微圆弧刃金刚石车刀切削刃的制造大部 分仍然是由熟练技工手工操作或半手工操作来完 成。加工过程中, 为保证切削刃的刃型精度需要利 用显微镜、投影仪以及非接触形状测定装置等进行 离线测量, 这种加工方式使切削刃研磨质量极易受 操作者技能水平、经验等主观因素影响, 同时研磨 过程中多次的离线测量使得加工过程无法连续进 行, 车刀每次重新装夹容易形成二次装夹误差。而 且仅靠技工的手工操作和肉眼观察很难准确地实现 对切削刃轮廓的针对性修整。很难保证被制造的金 刚石车刀具有稳定的刃型精度, 生产效率较低, 严 重阻碍了相关产业的进一步发展 ${ }^{[11]}$ 。

针对以上问题，本文提出了基于在线图像测量 的圆弧刃金刚石车刀自动研磨方法。利用在线图像 测量方法代替传统制造方法中的离线测量, 将在线 获取的切削刃的形状信息和位置信息反馈给控制系 统, 驱动研磨系统带动金刚石车刀按照最优的加工 策略自动有针对性地进行研磨和修整, 消除由单晶 金刚石各向异性造成的刃型误差。在这个方法中, 金刚石车刀切削刃的在线测量起着至关重要的作 用, 它决定了金刚石车刀切削刃轮廓信息(形状误 差、位置信息)的获取精度和速度, 进而影响着后续 的金刚石车刀切削刀的制造精度和效率。本文针对 金刚车刀研磨过程中的在线图像测量方法进行研 究, 确定获取金刚石车刀切削刃圆弧中心及研磨系 统的旋转中心的最佳图像处理方法, 在初始安装时 实时进行二者间的距离, 消除初始安装误差切削刃 加工精度及效率的影响, 并开发适用于微圆弧刃金 刚石车刀测量的图像测量系统, 实现微圆弧刃金刚 石车刀高效高精度在线测量。

\section{1 圆弧刃金刚石车刀创成原理及系统}

根据几何原理, 金刚石车刀圆弧刃可以由研磨 盘直线包络车刀围绕圆弧中心的旋转运动形成。同 时, 为控制车刀圆弧半径 $r$, 并对其施加稳定的研 磨压力, 还需要保证研磨车刀过程中有直线进给运 动功能。圆弧刃的创成原理如图 1 所示。研磨时, 无论车刀前端的形状如何, 最终所形成的圆弧状切 削刃的中心 $\left(x_{\mathrm{a}}, y_{\mathrm{a}}\right)$ 一定是和系统的摆动中心 $\left(x_{0}, y_{0}\right)$ 重合的。切削刃圆弧半径 $r$ 由车刀进给的长度来控 制, 研磨形成的切削刃上任一点 $\left(x_{i}, y_{i}\right)$ 距理想圆弧 中心即系统的摆动中心的距离 $r_{i}$ 可以由式(1)表示

$$
r_{i}=\sqrt{\left(x_{i}-x_{0}\right)^{2}+\left(y_{i}-y_{0}\right)}
$$

该点的位置可以用该点和车刀圆弧中点连线 与切削刃圆弧中点公法线 $O O^{\prime}$ 形成的角度 $\theta_{i}$ 来表 示，由式(2)计算

$$
\theta_{i}=\arctan \frac{x_{i}}{y_{i}}
$$

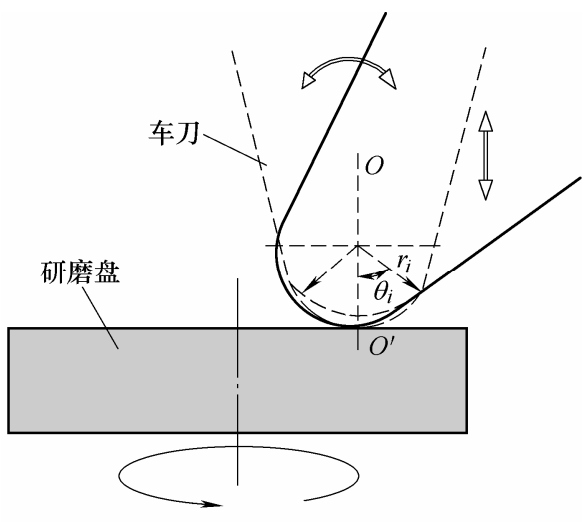

图 1 金刚石车刀圆弧切削刃创成原理图

由于金刚石的各向异性，在相同的压力作用下 切削刃上的不同点处的去除量不同，因此造成成形 后的切削刃上不同点处的 $r_{i}$ 也不同，形成了圆弧切 削刃的形状误差。该误差值 $\Delta r_{i}$ 可以由式(3)得到

$$
\Delta r_{i}=r_{i}-r
$$

由以上分析可知，如果能够在线测得圆弧切削 刃上各点的位置 $\theta_{i}$ 和 $r_{i}$ (或者 $\Delta r_{i}$ ) 值, 那么在研磨过 程中通过控制不同点处的研磨压力和驻留时间就可 以实现对切削刃有针对的研磨，从而提高圆弧切削 刃的形状精度。

因此，提出基于在线图像测量的圆弧刃金刚石 车刀研磨方法 ${ }^{[1]}$ 。该方法如图 2 所示。车刀被固定 在旋转工作台上, 旋转工作台被固定在垂直进给工 作台上，通过进给工作台的进给可以调节车刀与研 
磨盘接触的压力和车刀切削刃圆弧半径。在金刚石 车刀相对侧安装有 $\mathrm{CCD}$ 相机, 利用 $\mathrm{CCD}$ 相机可以 将车刀图像在线采集到计算机中, 通过一系列的图 像处理后能够获得车刀切削刃上各点的形状信息 $\left(\Delta r_{i}\right)$ 和位置信息 $\left(\theta_{i}\right)$, 将这些信息反馈给控制系统 后, 就可以通过控制研磨压力和驻留时间, 对切削 刃上去除残余或者崩坏部分进行有针对的研磨, 弥 补由于单晶金刚石各向异性造成的去除量不均产生 的误差。同时, 由于实现了在位自动测量, 避免了 传统的依靠手工操作、肉眼观察测量的缺点, 排除 了人为主观因素的干扰。

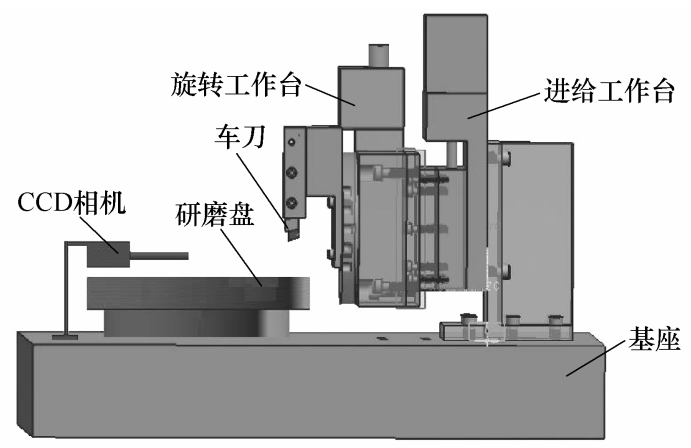

图 2 基于在线图像测量金刚石车刀研磨方法概念图

根据金刚石车刀圆弧刃创成原理可知，圆弧切 削刃的成形研磨由旋转工作台带动车刀摆动完成。 车刀初始安装时存在着研磨系统旋转中心和车刀切 削刃圆弧中心两个中心。车刀初始安装时, 车刀圆 弧圆心和系统旋转中心如果不一致, 就会形成初始 安装误差。初始安装误差过大, 会直接影响到圆弧 刀金刚石车刀切削刃形状精度和加工效率, 严重时 甚至会导致无法形成正确的切削刃形状。

\section{2 基于在线图像识别的装夹误差消除}

\section{1 金刚石车刀切削刃圆弧中心的获取}

通过上述分析可知, 在圆弧刃金刚石车刀研磨 开始前, 切削刃的圆弧中心 $\left(x_{\mathrm{a}}, y_{\mathrm{a}}\right)$ 和研磨系统的旋 转中心 $\left(x_{0}, y_{0}\right)$ 间的安装误差会直接影响到车刀的研 磨效率和精度, 甚至会导致无法形成正确的切削刃 型。因此必须在车刀初始装夹时尽量使两个中心重 合, 以保证在最小的研磨去除量的前提下获得最高 精度的圆弧切削刃。当垂直进给工作台固定不动时, 系统的摆动中心是不变的, 因此, 为使两个中心达 到重合, 只要在安装的时候调整车刀, 使车刀的切 削刃圆弧中心靠近系统旋转中心, 并最终使二者重 合。这就需要图像测量系统能够快速准确地捕捉到 切削刃的圆弧中心。
获得金刚石车刀切削刃的圆弧中心, 可以通过 三种图像处理方法实现：(1) 圆弧拟合法，对 CCD 采集的每帧图像进行一系列的图像预处理(平滑、二 值化)后, 利用 Laplace 算子考察图像的每个像素在 某个邻域内灰度的变化, 利用边缘邻近一阶或二阶 方向导数变化规律来提取金刚石车刀图像的轮廓, 由于提取处的轮廓包括了两侧棱线(非切削刃)和前 端的圆弧线(切削刃), 根据 Ramer 算法对提取到的 轮廓进行递归细分, 并将其分割为圆弧和直线两部 分, 并求得构成圆弧的点群坐标值, 利用这些点群 信息，基于最小二乘法进行圆弧拟合，得到圆弧部 分在图像坐标系内的圆心坐标; (2) 形状模板匹配 法, 在首次采集到的金刚石车刀图像上, 基于 Sobel 等边缘提取算子提取出车刀前端轮廓，将车刀圆弧 切削刃轮廓部分的点群及方向矢量信息建立模板, 利用此模板对 $\mathrm{CCD}$ 后续采集到的金刚石车刀图像 进行匹配, 从而得到车刀切削刃圆弧中心在图像坐 标系的坐标值; (3) 灰度值模板匹配法, 此方法和形 状模板匹配法类似，将首次采集到的图像指定区域 的灰度分布作为模板, 和后续循环采集到的图像进 行匹配，从而得到金刚石车刀切削刃圆弧中心。

分别利用以上三种方法对同一金刚石车刀图 像进行切削刃圆弧中心识别, 比较三种方法的实时 性。各方法识别所消耗的时间如图 3 所示。

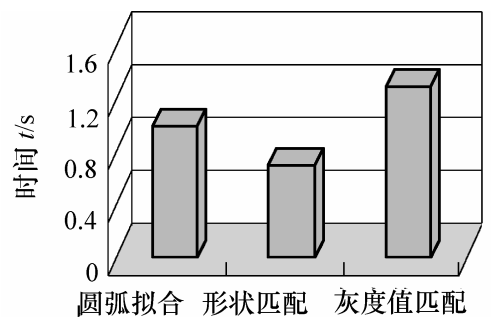

图 3 金刚石车刀切削刃圆弧中心识别时间

从图 3 中可以看出, 实时性最差的是基于灰度 值的模板匹配方法, 测量效率为 $1.3 \mathrm{~s}$; 基于形状的 模板匹配方法实时性最好, 可以达到 $0.7 \mathrm{~s}$ 的测量效 率，仅为基于灰度值匹配方法的二分之一左右。

图像识别系统对金刚石车刀切削刃圆弧中心 的获取不仅要迅速, 而且还要准确, 否则容易造成 装夹误差增大。图像识别误差主要是由于光源的非 稳定照射及周围环境因素变化造成的。为了比较三 种方法获取车刀切削刀圆弧中心的准确性，分别利 用三种图像识别方法对静止不动的金刚石车刀进行 1000 次的切削刃圆弧中心提取, 并计算获得的圆弧 中心坐标 $x_{\mathrm{a}} 、 y_{\mathrm{a}}$ 的方差, 分别如图 4 所示。 


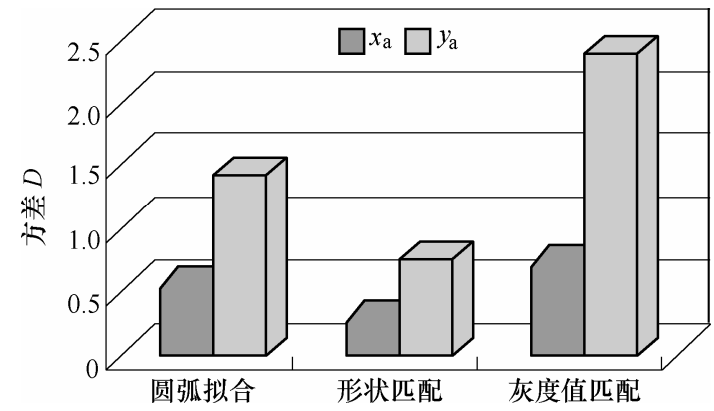

图 4 不同方法获取切削刃圆弧中心方差

通过对方差的计算发现, 形状模板匹配法获取 的切削刃圆弧中心无论是坐标 $x_{\mathrm{a}}$ 还是坐标 $y_{\mathrm{a}}$ 的方差 都要远小于其他两种方法, 表明这种方法测得结果 的平均偏离程度较小, 数据的波动性最小, 因此测 量的稳定性最高。进一步统计获取切削刃圆弧中心 落入误差范围的次数, 如表 1 所示。从表 1 中可以 看出, 基于形状的模板匹配方法 $x_{\mathrm{a}} 、 y_{\mathrm{a}} 、\left(x_{\mathrm{a}}, y_{\mathrm{a}}\right)$ 在小 于等于 $\pm 0.5 \mu \mathrm{m}$ 范围内的次数分别为 $616,406,255$, 在小于 $\pm 1.0 \mu \mathrm{m}$ 范围内次数分别为 $933,768,730$, 均多于其他两种方法; 而在大于等于 $\pm 1.0 \mu \mathrm{m}$ 范围 内的次数分别为 $67,232,27$, 远少于其他两种图 像识别方法, 可见基于形状模板匹配法获得的切削 刃圆弧中心准确性和精确性最高, 受环境变化因素 影响最小, 其鲁棒性要优于其他两种图像识别方法。 因此本研究采用形状模板匹配法进行圆弧刃金刚石 车刀圆心的获取。

表 1 不同误差带内三种方法获取坐标统计

\begin{tabular}{clccc}
\hline $\begin{array}{c}\text { 误差范围/ } \\
\mu \mathrm{m}\end{array}$ & 比较点 & $\begin{array}{c}\text { 圆弧拟 } \\
\text { 合法 }\end{array}$ & $\begin{array}{c}\text { 形状模板匹 } \\
\text { 配法 }\end{array}$ & $\begin{array}{c}\text { 灰度值模 } \\
\text { 板匹配法 }\end{array}$ \\
\hline \multirow{3}{*}{$-0.5 \leqslant \delta \leqslant 0.5$} & 坐标 $x_{\mathrm{a}}$ & 406 & 616 & 457 \\
& 坐标 $y_{\mathrm{a}}$ & 355 & 406 & 258 \\
& 坐标 $\left(x_{\mathrm{a}}, y_{\mathrm{a}}\right)$ & 146 & 255 & 144 \\
\hline \multirow{3}{*}{$-1.0 \leqslant \delta \leqslant 1.0$} & 坐标 $x_{\mathrm{a}}$ & 818 & 933 & 795 \\
& 坐标 $y_{\mathrm{a}}$ & 606 & 768 & 526 \\
& 坐标 $\left(x_{\mathrm{a}}, y_{\mathrm{a}}\right)$ & 535 & 730 & 452 \\
\hline \multirow{3}{*}{$|\delta|>1.0$} & 坐标 $x_{\mathrm{a}}$ & 182 & 67 & 205 \\
& 坐标 $y_{\mathrm{a}}$ & 394 & 232 & 474 \\
& 坐标 $\left(x_{\mathrm{a}}, y_{\mathrm{a}}\right)$ & 165 & 27 & 148 \\
\hline
\end{tabular}

\section{2 研磨系统的旋转中心的获取}

实现了金刚石车刀切削刃圆弧中心的在线获 取, 那么就可以根据获取的切削刃圆弧中心坐标计 算其和研磨系统旋转中心的距离, 并调节车刀位置 使其切削刃圆弧中心和系统旋转中心重合。由于获 取的切削刃圆弧中心坐标是在 $\mathrm{CCD}$ 的图像坐标系 内, 为了便于计算还需要得到系统旋转中心在图像 坐标系内的坐标。根据金刚石车刀圆弧刃创成原理, 研磨过程中研磨系统的摆动中心是固定的, 因此,
根据几何学原理, 只要装夹时金刚石车刀上任意有 一点和系统旋转中心重合时, 那么当系统带动金刚 石旋转时, 金刚石前刀面上包括切削刃圆弧中心在 内的所有点都会围绕系统旋转中心做旋转运动, 即 切削刃圆弧中心的轨迹是以系统旋转中心为圆心的 一段圆弧。因此只要能够获得车刀上切削刃圆弧中 心在摆动过程中在不同位置的坐标, 并通过最小二 乘法进行圆弧拟合即可获得系统的旋转中心。本研 究中, 将车刀安装在夹具上在 $-60^{\circ}$ 到 $60^{\circ}$ 范围内摆 动, 并且每旋转 $5^{\circ}$, 利用前述形状模板匹配法获取 车刀切削刃圆弧中心 $\left(x_{\mathrm{a}}, y_{\mathrm{a}}\right)$, 具体数值如表 2 所示。 利用获取的不同位置切削刃圆弧中心进行圆弧拟 合, 可以得到切削刃圆弧中心摆动轨迹的圆心坐标 为(752.377，436.811), 如图 5 所示。根据前述分析 可知, 此坐标即为研磨系统旋转中心在图像坐标系 中的位置。

表 2 不同旋转角度获取的切削刃圆弧中心坐标

\begin{tabular}{cc||cc}
\hline 旋转角 & $\left(x_{\mathrm{a}}, y_{\mathrm{a}}\right)$ & $\begin{array}{c}\text { 旋转角度 } \\
\theta /\left(^{\circ}\right)\end{array}$ & $\left(x_{\mathrm{a}}, y_{\mathrm{a}}\right)$ \\
\hline 度 $\theta /\left(^{\circ}\right)$ & $(709.257,508.914)$ & 5 & $(715.056,514.711)$ \\
\hline 0 & $(702.852,505.245)$ & 10 & $(719.616,516.345)$ \\
-5 & $(694.261,496.888)$ & 15 & $(719.088,514.963)$ \\
-10 & $(686.147,491.718)$ & 20 & $(727.813,519.113)$ \\
-20 & $(681.104,485.127)$ & 25 & $(732.240,524.000)$ \\
-25 & $(676.774,477.990)$ & 30 & $(741.652,525.511)$ \\
-30 & $(677.304,473.933)$ & 35 & $(747.268,524.713)$ \\
-35 & $(675.294,466.945)$ & 40 & $(754.597,521.975)$ \\
-40 & $(672.750,458.715)$ & 45 & $(761.780,523.162)$ \\
-45 & $(669.723,451.182)$ & 50 & $(770.485,521.265)$ \\
-50 & $(667.493,439.276)$ & 55 & $(771.515,517.596)$ \\
-55 & $(663.383,431.491)$ & 60 & $(779.215,515.635)$ \\
-60 & $(662.544,419.297)$ & & \\
\hline
\end{tabular}

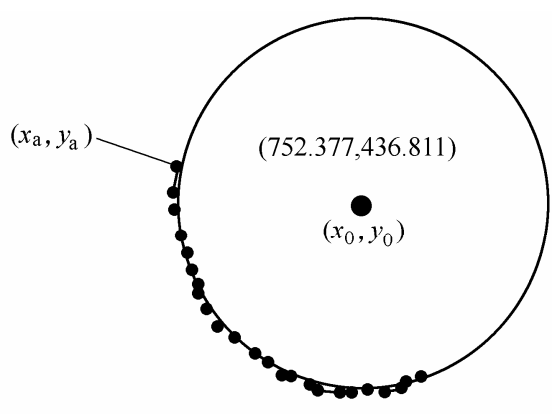

图 5 研磨系统旋转中心获取

确定研磨系统的旋转中心后, 就可以在金刚石 车刀的初始安装时对车刀的位置进行在线调整, 消 除初始安装误差对车刀加工精度的影响。在安装过 程中始终在线获取切削刃圆弧中心位置 $\left(x_{\mathrm{a}}, y_{\mathrm{a}}\right)$, 并 计算其和研磨系统旋转中心间的距离, 通过夹具上 
的调整装置对车刀位置进行调整, 直至切削刃圆弧 中心和研磨系统旋转中心重合, 如图 6 所示。

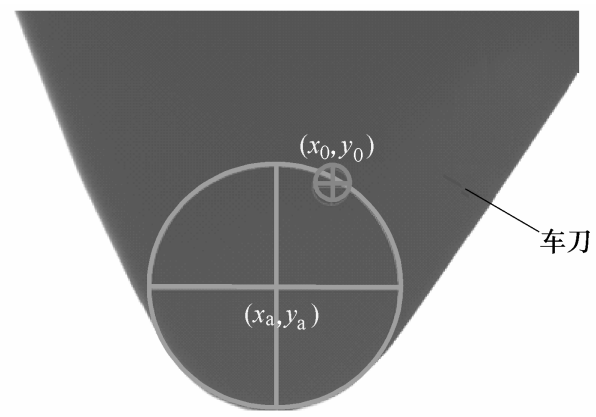

(a) 初始安装中心调整前

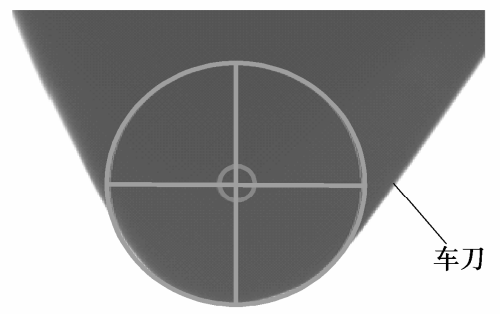

(b) 初始安装中心调整后

图 6 金刚石车刀初始安装中心调整

\section{3 切削刃轮廓度误差在线图像测量}

传统的金刚石车刀研磨过程采用车刀恒速摆 动和恒定进给量的加工方式, 由于单晶金刚石具有 各向异性, 不同方向的硬度差异很大, 因此在相同 加工工艺条件下, 在切削刃不同点处的材料去除量 势必会有差异, 从而形成了切削刃轮廓度误差。本 研究基于在线图像测量获取的切削刃误差信息, 研 磨过程中对切削刃不同误差位置以不同的驻留时间 或者进给量进行针对性研磨, 可以有效地减小由单 晶金刚石的各向异性导致的轮廓度误差, 并提高加 工效率。

为实现针对性研磨, 切削刃上的各点误差信息 及其对应的位置信息是必须的。如图 7 所示, 通过 对 CCD 采集到的金刚石车刀图像在指定区域内进 行二值化、平滑等一系列处理后, 提取车刀图像轮 廓, 并通过 Ramer 算法将切削刃的轮廓线(圆弧部 分)分离出来。利用式(1) (3)即可计算出构成切削 刃轮廓线的点群与理想切削刃轮廓线的对应点之间 的误差及其对应位置。其流程如图 7 所示。根据获 得误差及其位置信息, 研磨系统在粗加工阶段采取 进给量正比于误差大小, 在精加工阶段采取车刀摆 动速度反比于误差大小的不同控制策略对切削刃进
行针对性研磨。

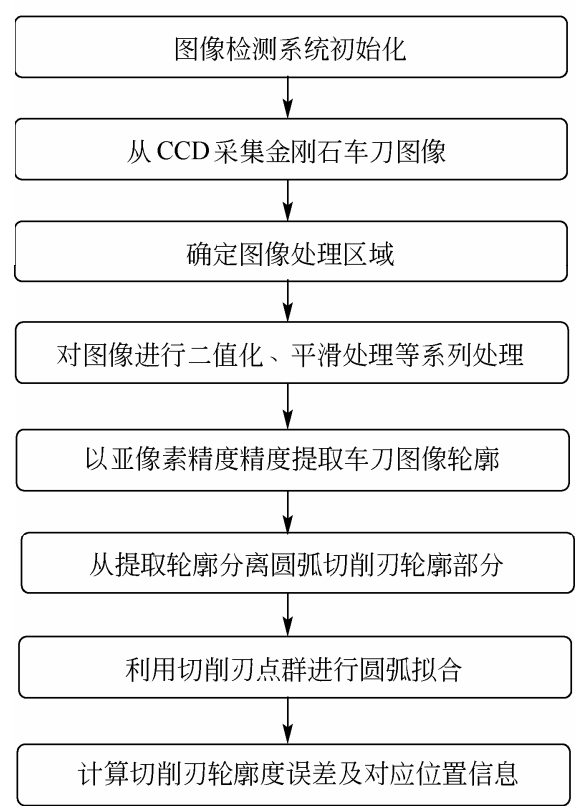

图 7 图像检测切削刃轮廓度误差的流程图

为了验证微圆弧刃金刚石车刀切削刃在线图 像检测方法的精度，分别采用在线图像检测方法和 接触式扫描的方法对同一把车刀进行了切削刃轮廓 度误差检测。接触式扫描方法是传统金刚石车刀研 磨过程中进行离线测量的主要方法之一, 通过扫描 车刀后刀面靠近切削刀的轮廓得到切削刃轮廓度误 差。本文采用 Talysurf PGI 1250 轮廓仪对切削刃形 状进行扫描, 扫描时调节探针在车刀后刀面位置, 使其尽量靠近切削刃，沿着切削刃圆弧方向进行扫 描, 利用扫描获得的测量点群坐标基于最小二乘法 进行圆弧拟合，从而得到圆弧中心，利用式(1) (3) 计算, 可以获得各测量点的轮廓度误差及其对应位 置。如图 8 所示, 图 8 中曲线 1 为开发的在线图像 测量方法得到切削刃轮廓度误差曲线, 曲线 2 为扫 描最靠近切削刃的后刀面得到轮廓度误差曲线。比 较两条曲线可以发现，两条曲线从整体变化趋势和 细节部分都非常类似, 最大误差都出现在 $0^{\circ}$ 左右, 这说明本研究所开发的在线图像测量系统能够准确 得到切削刃轮廓度误差及其分布。但是从图 8 中也 可以看出, 虽然两条曲线的变化趋势几乎相同, 但 轮廓度误差大小却存在着约 $0.45 \mu \mathrm{m}$ 的差距。为了 分析两种测量方法出现差距的原因, 在后刀面的不 同位置分别进行了轮廓扫描, 并计算其轮廓度误差, 分别为图 8 中曲线 3 5, 曲线标号越大, 探针扫描 的位置越远离切削刃。比较图 8 中的轮廓度误差曲 线 2 5 可知, 随着扫描位置越远离切削刃, 轮廓仪 测量得到的轮廓度误差差距越大，曲线 $4 、 5$ 甚至曲 线的形状(变化趋势)也和曲线 $2 、 3$ 产生了很大差异。 
这主要是由于研磨过程中对后刀面不同位置研磨效 果偏差造成的, 越远离切削刃, 研磨效果越差, 轮 廓度误差也会越大。由于车刀后角的存在, 实际上 圆弧刀金刚石车刀的后刀面是一个圆雉体。探针扫 描测量的只是后刀面, 很难准确定位在切削刃上, 如图 9 所示。因此探针扫描测量时测量的只是车刀 后刀面, 而非真正切削刀轮廓。而对于图像测量来 说, 测量时 $\mathrm{CCD}$ 相机镜头的轴线垂直车刀前刀面, 摄取车刀前刀面的图像的轮廓线才真正是车刀的切 削刃。由于两种测量方法测量的位置不同, 测量结 果也必然会存在误差。从测量原理来看, 相比于传 统探针扫描测量方法, 在线图像测量的结果应该更 接近于切削刃的实际轮廓度。

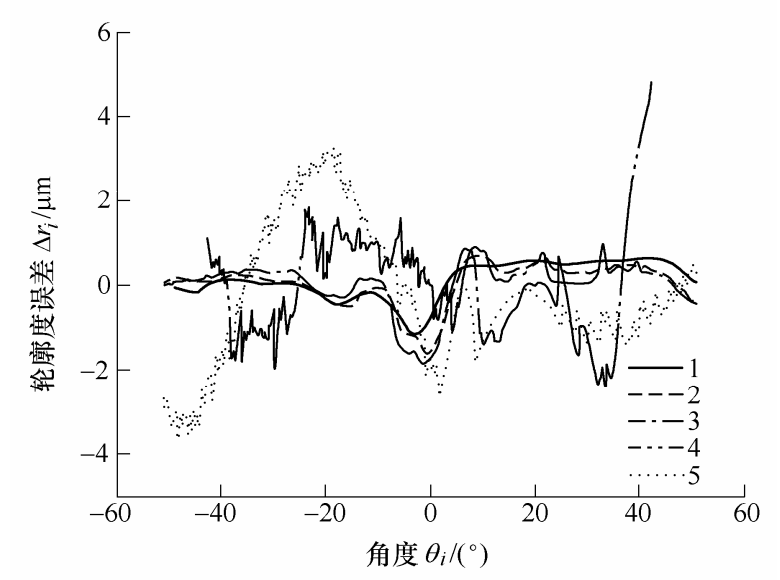

图 8 切削刃轮廓度测量结果

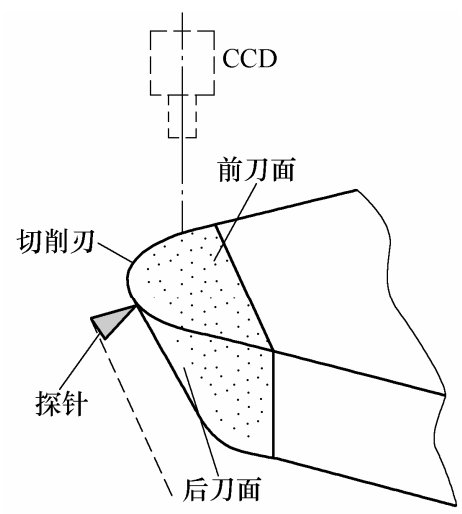

图 9 在线图像测量法与接触式扫描法测量原理

\section{4 结论}

(1) 在圆弧刃金刚石车刀自动研磨系统的在线 识别方法中, 形状模板匹配方法在识别效率和精确 性上要远优于灰度值模板匹配和直接圆弧拟合方 法，更适用于圆弧刀金刚石车刀在线测量。
(2) 利用在线获取金刚石车刀切削刀圆弧中心 及实时计算其和研磨系统的旋转中心距离, 实现了 初始装夹时金刚石车刀位置的实时调整, 从而消除 了初始安装误差对切削刃加工精度及效率的影响。

(3) 开发了适用于微圆弧刃金刚石车刀测量的 图像测量系统, 实际测量验证试验表明, 从原理上 利用开发的在线图像测量方法获得的切削刃轮廓信 息更准确, 更适合圆弧刀金刚石车刀高效、高精度、 低成本制造。

\section{参 考 文 献}

[1] ZHAO Qingliang, WANG Yilong, YU Guang, et al. Investigation of anisotropic mechanisms in ultra-precision diamond machining of KDP crystal[J]. Journal of Materials Processing Technology, 2009, 209(9) : 4169-4177.

[2] 袁巨龙, 王志伟, 文东辉, 等. 超精密加工现状综述 [J]. 机械工程学报, 2007, 43(1): 35-48.

YUAN Julong, WANG Zhiwei, WEN Donghui, et al. Review on ultra-precision machining technology[J]. Chinese Journal of Mechanical Engineering, 2007, 43(1): $35-48$.

[3] GAO Wei, LEE J C, ARAI Y, et al. Measurement of slide error of an ultra-precision diamond turning machine by using a rotating cylinder workpiece[J]. International Journal of Machine Tools and Manufacture, 2010, 50(4): 404-410.

[4] FANG Fengzhou, ZHANG Xiaodong, HU Xiaotang. Cylindrical coordinate machining of optical freeform surfaces[J]. Optics Express, 2008, 16(10): 7323-7329.

[5] WANG Yilong, ZHAO Qingliang, SHANG Yuanjiang, et al. Ultra-precision machining of Fresnel microstructure on die steel using single crystal diamond tool[J]. Journal of Materials Processing Technology, 2011, 211(12): 2152-2159.

[6] ZONG Wenjun, SUN Tao, LI Dan, et al. Nano-precision diamond cutting tools achieved by mechanical lapping versus thermo-mechanical lapping[J]. Diamond \& Related Materials, 2008, 17: 954-961.

[7] BIDDUT A Q, RAHMAN M, NEO K S, et al. Performance of single crystal diamond tools with different rake angles during micro-grooving on electroless nickel plated die materials[J]. Int. J. Adv. Manuf. Technol., 2007, 33: 891-899.

[8] QIU Zhongjun, FANG Fengzhou, DING Liyu, et al. Investigation of diamond cutting tool lapping system 
based on on-machine image measurement[J]. Int. J. Adv.

Manuf. Technol., 2011, 56: 79-86.

[9] 袁哲俊. 精密和超精密加工技术的新进展 [J]. 工具技 术，2006，40(8): 3-9.

YUAN Zhejun. New developments of precision and ultra-precision manufacturing technology[J]. Tool Engineering, 2006, 40(8): 3-9.

[10] ZONG Wenjun, LI Zengqiang, SUN Tao, et al. The basic issues in design and fabrication of diamond-cutting tools for ultra-precision and nano-metric machining[J]. Int. J.
Mach. Tools Manuf., 2010, 50: 411-419.

[11] QIU Zhongjun, ZHOU Libo, EDA H, et al. Development of vision guided grinding system for diamond turning tools[J]. Key Engineering Materials, 2008, 375-376: 705-709.

作者简介: 仇中军, 男, 1974 年出生, 博士, 副教授, 硕士研究生导师。 主要研究方向为超精密加工、微纳制造工艺及装备。

E-mail: qiuzhongjun@gmail.com

房丰洲(通信作者), 男, 1963 年出生, 博士, 教授, 博士研究生导师。 主要研究方向为超精密加工理论及工艺、微纳制造技术及装备。

E-mail: fzfang@tju.edu.cn

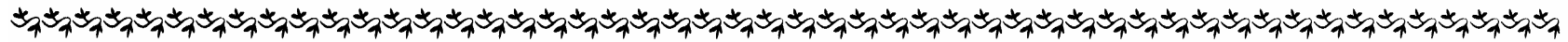

\section{第三届 IFToMM 亚洲机构与机器科学会议征文通知}

为增进亚洲地区机构与机器科学及其相关学科研究人员、行业专家以及学生间的学术交流, 第三届 IFToMM 机构与机 器科学会议于 2014 年 7 月 9-10 日在中国天津召开。

本次会议由天津大学和中国机械工程学会共同主办, IFToMM 中国委员会承办。会议将围绕机构与机器科学等议题开展 讨论, 主要包括理论运动学、计算运动学、机器零件、驱动器/执行机构、齿轮及传动机构、连杆与凸轮、机构设计、机械 动力学、摩擦学、车辆机构、动力学与设计、可靠性、机构试验方法、机器人及机电一体化、生物力学、微纳机构与机器、 医疗/保健设备、自然与机器、设计方法、机构与机器科学史、机构与机器科学教育、可重构机构、折纸灵感机构。

请按照会议网站论文要求准备稿件, 并将 PDF 版本上传至会议网站 http://www.asianmms2014.com。会议组委会将组织 同行专家进行全文评审。

全文提交截止日期：2014 年 2 月 10 日

录用通知日期：2014 年 3 月 31 日

修改论文提交截止日期： 2014 年 4 月 30 日 